BMC

Developmental Biology

Retinoic acid is a key regulatory switch determining the difference between lung and thyroid fates in Xenopus laevis

Wang et al. 


\title{
Retinoic acid is a key regulatory switch determining the difference between lung and thyroid fates in Xenopus laevis
}

Jean H Wang ${ }^{1}$, Steven J Deimling ${ }^{1,2}$, Nicole E D'Alessandro ${ }^{1,2}$, Lin Zhao ${ }^{1,3}$, Fred Possmayer ${ }^{1,3,4}$ and Thomas A Drysdale $e^{1,2,5^{*}}$

\begin{abstract}
Background: The lung and thyroid are derived from the anterior endoderm. Retinoic acid and Fgf signalling are known to be essential for development of the lung in mouse but little is known on how the lung and thyroid are specified in Xenopus.

Results: If either retinoic acid or Fgf signalling is inhibited, there is no differentiation of the lung as assayed by expression of sftpb. There is no change in expression of thyroid gland markers when retinoic acid signalling is blocked after gastrulation and when Fgf signalling is inhibited there is a short window of time where pax 2 expression is inhibited but expression of other markers is unaffected. If exogenous retinoic acid is given to the embryo between embryonic stages 20 and 26, the presumptive thyroid expresses sftpb and sftpc, specific markers of lung differentiation and expression of key thyroid transcription factors is lost. When the presumptive thyroid is transplanted into the posterior embryo, it also expresses sftpb, although pax2 expression is not blocked.

Conclusions: After gastrulation, retinoic acid is required for lung but not thyroid differentiation in Xenopus while Fgf signalling is needed for lung but only for early expression of pax 2 in the thyroid. Exposure to retinoic acid can cause the presumptive thyroid to switch to a lung developmental program.
\end{abstract}

\section{Background}

The differentiation of specific organs from the endoderm is directly related to their position along the anteroposterior axis of the vertebrate embryo. After initial specification of the endoderm, wnt, nodal, bone morphogenetic protein and fibroblast growth factor (Fgf) signals quickly subdivide the gut into the foregut, midgut and hindgut [1]. Expression of hhex, sox2 and foxa 2 are associated with the foregut [2] whereas expression of sox 17 [3] and $c d x$ [4] genes is eventually restricted to posterior endoderm. The foregut will give rise to the thyroid, thymus, parathyroid, lung, liver, pancreas, and stomach.

Although specific transcription factors are associated with each of these organ systems in later development, expression of key transcription factors at early stages is

\footnotetext{
* Correspondence: tadrysda@uwo.ca

${ }^{1}$ Children's Health Research Institute, London, Ontario, Canada

Full list of author information is available at the end of the article
}

not restricted to a specific organ system. In the foregut, $n k x 2.1$ (or ttf1) is required for development of both the lung and thyroid suggesting a close relationship between these tissues but each organ has a distinct set of transcription factors that are required for differentiation. The thyroid also expresses hhex, pax8, and foxe1 [5], although in Xenopus and zebrafish, pax2 is the Pax gene expressed in the thyroid [6,7]. In addition to $n k x 2.1$, the lung expresses foxa2 and foxp 2 [8-10]. The interplay of many different signalling pathways is necessary for defining both of these organs. Fibroblast growth factor (Fgf) signalling has been shown to be essential for both thyroid [11] and lung although the signal amplitude and timing are important for determining the output from the Fgf signal [12].

Recently, in addition to Fgf signalling, it has been demonstrated that graded levels of retinoic acid (RA) are important in chick for defining the different regions along the anterior-posterior axis of the developing endoderm [13]. The most anterior endoderm, that gives rise
C Biomed Central

(c) 2011 Wang et al; licensee BioMed Central Ltd. This is an Open Access article distributed under the terms of the Creative Commons Attribution License (http://creativecommons.org/licenses/by/2.0), which permits unrestricted use, distribution, and reproduction in any medium, provided the original work is properly cited. 
to the thyroid, forms with little or no RA although RA is necessary for other anterior endoderm derivatives including the lung $[14,15]$ and pancreas $[16,17]$. In frog, aldh1a2 (raldh2), encoding the enzyme primarily responsible for the synthesis of RA, is expressed dynamically throughout development. After gastrulation when patterning of the gut is being established, expression is found in the lateral plate mesoderm overlying the anterior gut with highest levels on the dorsal side. There are discrete regions of expression in the head but on the ventral side, there is little expression anterior to the forming heart $[18,19]$. This would predict that the forming lung should be exposed to RA but that more posterior endoderm would be exposed to lower levels of RA. The most anterior endoderm, giving rise to the thyroid, should also have limited exposure to RA. Later in development, expression of aldh1a2 is found in specific gut regions where it is required for the gut looping process [20].

In Xenopus, differentiation markers that can be used to study development of the lung have only recently been identified [21]. In that study, surfactant protein $B$ $(s f t p b)$ and surfactant protein $C$ (sftpc) were shown to have high sequence conservation with other homologues and were surprisingly early markers of lung in Xenopus, being expressed as early as stage 39. Sftpb and $s f t p c$ encode important constituents of pulmonary surfactant, a material that regulates surface tension in the lung alveoli and are expressed solely in the lung [22]. Although the role of thyroid hormone in Xenopus biology has been extensively studied [23], the development of the thyroid is not well characterized. Expression studies have demonstrated key early transcription factors, identified as essential in other organisms, likely play a role in thyroid development in Xenopus including $n k x 2.1$ [24], pax genes [6,25], foxe1, and foxe4 [25], but how the thyroid is specified in frog is not known.

The goal of this study was to test the hypothesis that the underlying mechanism for the development of the lung and thyroid in Xenopus is similar to other model organisms. In doing so, we demonstrate that the requirement for RA and Fgf signalling in lung differentiation is conserved in Xenopus. Furthermore, if exogenous retinoic acid is applied to the embryo after gastrulation, the thyroid primordium will express $s f t p b$ and sftpc. In addition, expression of early transcription factors that are normally expressed in the thyroid but not the lung, pax2, foxe 4 , and hhex are lost in presumptive thyroid in response to retinoic acid. These results further demonstrate that spatial control of specific signalling factors is essential for the patterning of the foregut endoderm and that retinoic acid signalling is a key element of that process.

\section{Results}

\section{Temporal expression of lung and thyroid markers}

To establish a baseline of when the different markers of lung and thyroid are first detectable, their expression was compared by whole mount in situ hybridization (Figure 1). Nkx2.1 expression was first detectable in the thyroid at stage 30 and appeared as a small dot immediately caudal to the cement gland. There was only a slight delay before expression of pax 2 was detected in the thyroid. At stage 34, expression of $n k x 2.1$ was detectable in the forming lung. As expected, the expression of these key transcription factors preceded the expression of the lung differentiation markers $s f t p b$ and $s f t p c$ both of which were detectable at stage 38 .

\section{Retinoic acid and FGF are required for lung development in Xenopus}

A requirement for both RA and Fgf signalling has been demonstrated in mouse lung development [26-29] and we wished to confirm that this requirement is conserved in Xenopus. Using a pan-retinoic acid receptor antagonist [30], we were able to show that there is a requirement for RA signalling. However, the ability of the antagonist to suppress lung differentiation, as assayed by $s f t p b$ expression is restricted to very early stages of lung development. If RA signalling was blocked at stage 26, well before the expression of $n k x 2.1$, lung differentiation occurred normally (Figure 2). Expression of $s f t p b$ was detectable in some embryos when RA signalling was blocked as early as stage 20, although most had no expression and those that did had lower expression levels than control embryos. These results are mirrored by the expression of $n k x 2.1$. If RA signalling is blocked prior to stage 20, $n k x 2.1$ expression is lost in the lung although maintained in the thyroid. At stage 20 there is lung expression in some embryos and if treatment with the RA antagonist is delayed until stage 26 , essentially all embryos express $n k x 2.1$ in the lung.

If Fgf signalling was blocked using the inhibitor SU5402 after stage 12,20 , or even stage 26 , expression of $n k \times 2.1$ was lost in the lung region whereas the expression in the forming thyroid was maintained (Figure 3). Again, as expected, blocking Fgf signalling also resulted in a loss of $s f t p b$ expression, although some embryos did show some very weak sttpb expression (Additional File 1, Figure S1). The loss of Fgf signalling was also confirmed by looking at the expression of sprouty2, a direct target of Fgf signalling (Additional File 1, Figure S1). In addition, expression of foxe4 was maintained in the forming thyroid in the presence of SU5402. This suggests that Fgf signalling was required for lung specification but not thyroid specification at the times tested. Expression of foxe 4 in the forming lens 


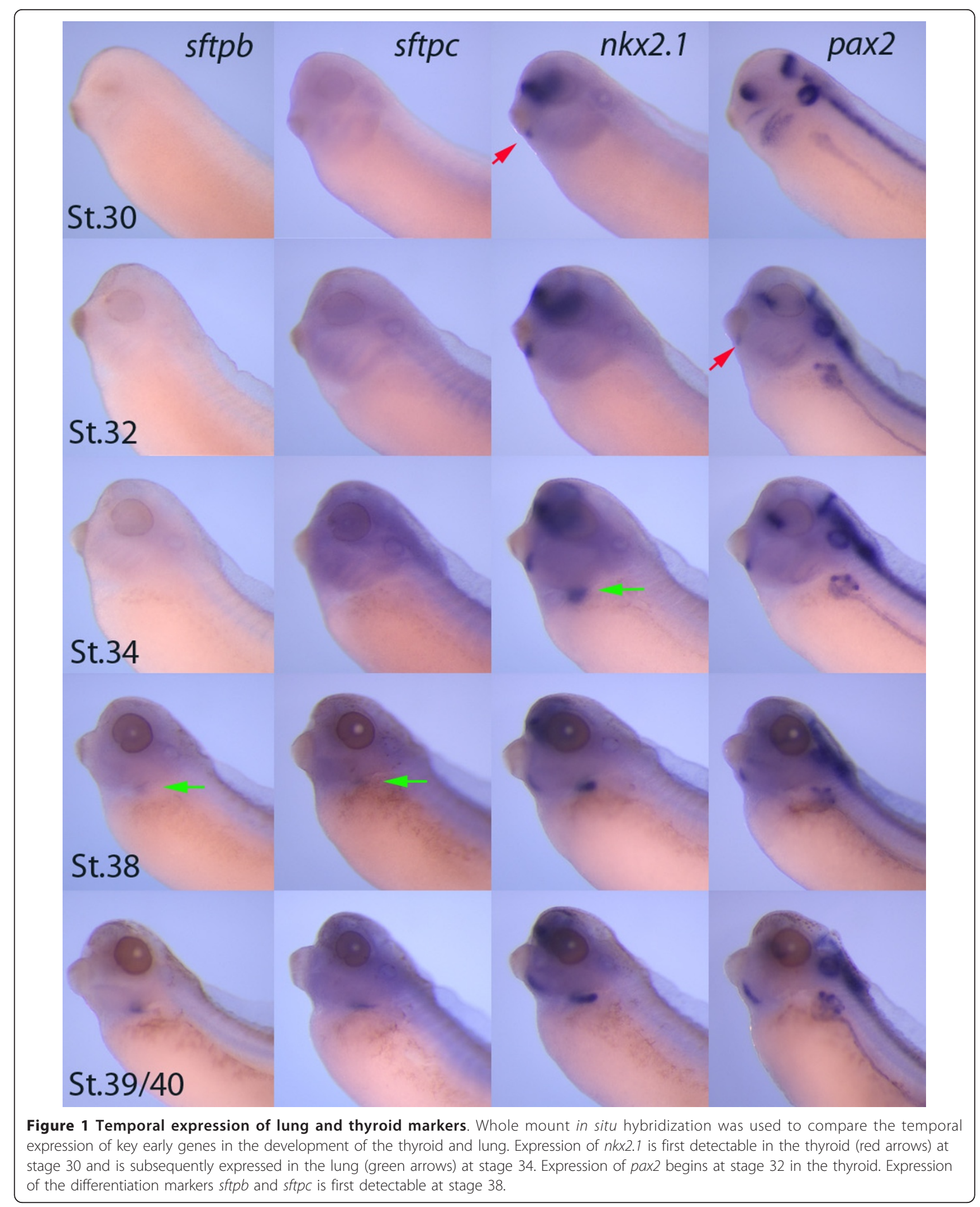




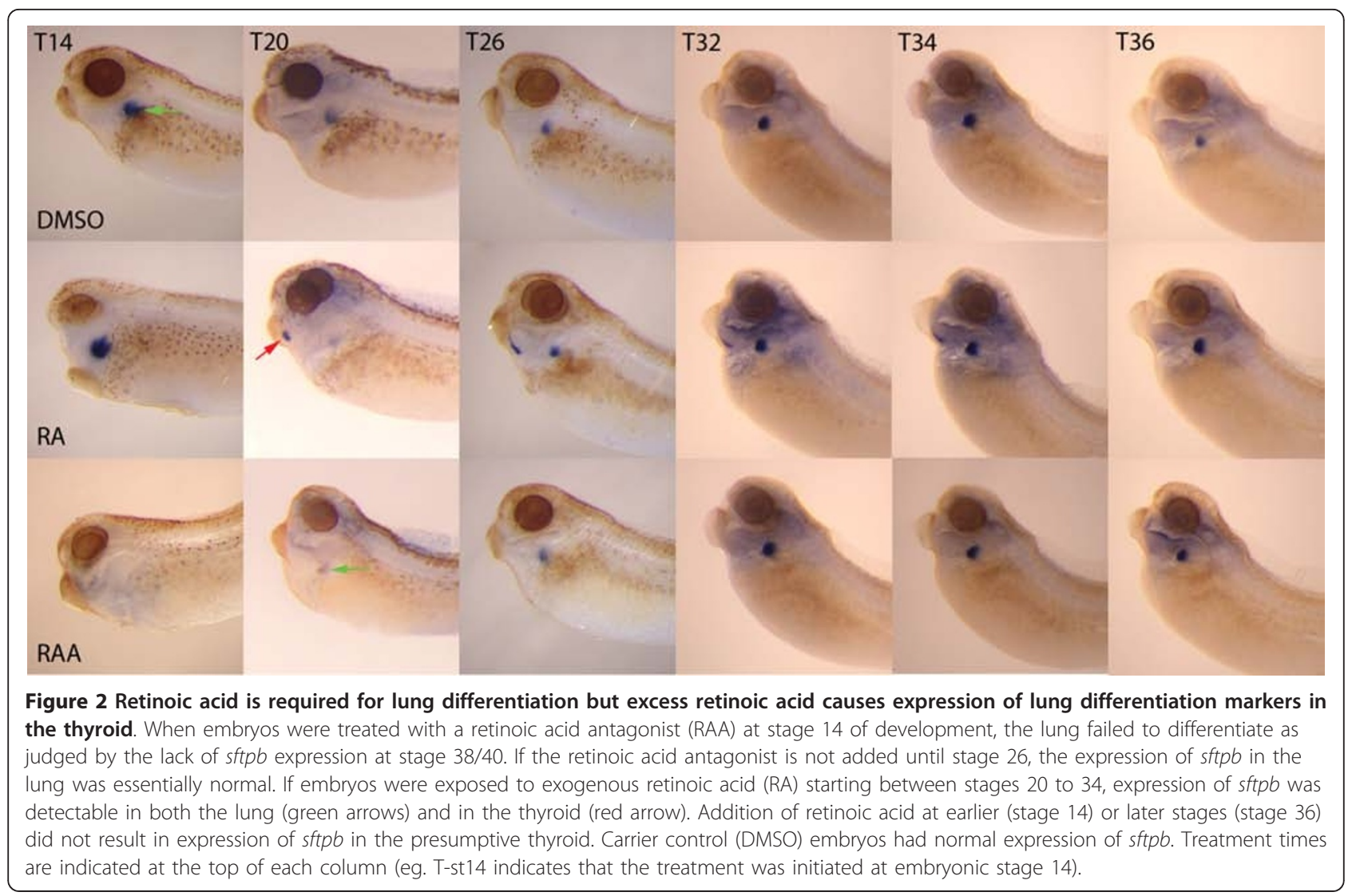

was maintained when FGF signalling was blocked but the lens morphology was aberrant when compared to controls in Fgf inhibitor treatments that began at stage 20 (Figure 3). Given that Fgf signalling has been implicated in thyroid development in other organisms [7], we also looked at the expression of the other early thyroid marker, pax2. We found that blocking Fgf signalling immediately after gastrulation (stage12) resulted in no thyroid expression of pax2, although expression in other tissues remained unaffected (Figure 3). When Fgf signalling was blocked at stage 20, pax2 expression in the thyroid was apparently normal, although expression was not observed in all embryos. Treatment at stage $26 \mathrm{had}$ no effect on pax2 expression in the thyroid.

\section{Exogenous retinoic acid causes the thyroid to express the lung developmental program}

As the requirement for RA was very early in the process of lung organogenesis, one potential role for retinoic acid could be to determine the number of lung progenitors. Therefore, we also treated embryos with exogenous retinoic acid to see if there was an increase in the size of the developing lung. Although we did not see any clear increase in lung size, there was ectopic expression of $s f t p b$ in a small region of the embryo that corresponded both in size and shape with the developing thyroid (Figure 2). Addition of RA was able to cause this ectopic expression even when retinoic acid was added as late as stage 34 and the activity was dose dependent (Table 1). Concentrations of RA that were able to cause ectopic expression of $s f t p b$ were the same as those that caused loss of pax 2 expression in the thyroid suggesting that the two processes might be linked (Table 1). When embryos were treated with RA at stage 14 , there was no ectopic expression of $s f t p b$ posterior to the cement gland (Figure 2) although there was also no expression of pax 2 or foxe 4 .

In order to determine if this simply represented ectopic expression of $s f t p b$ in the thyroid or a more global change towards lung differentiation in the thyroid, we examined expression of $s f t p c$, another highly specific lung specific marker and found that it was also expressed in the thyroid primordium when embryos were exposed to RA (Figure 4). The presence of $s f t p b$ and sftpc mRNA suggests either that RA causes lung differentiation markers to be expressed in the thyroid or that it causes the conversion of presumptive thyroid tissue into lung. To distinguish between these possibilities, we examined the expression of the key early transcription factors in lung and thyroid development in 


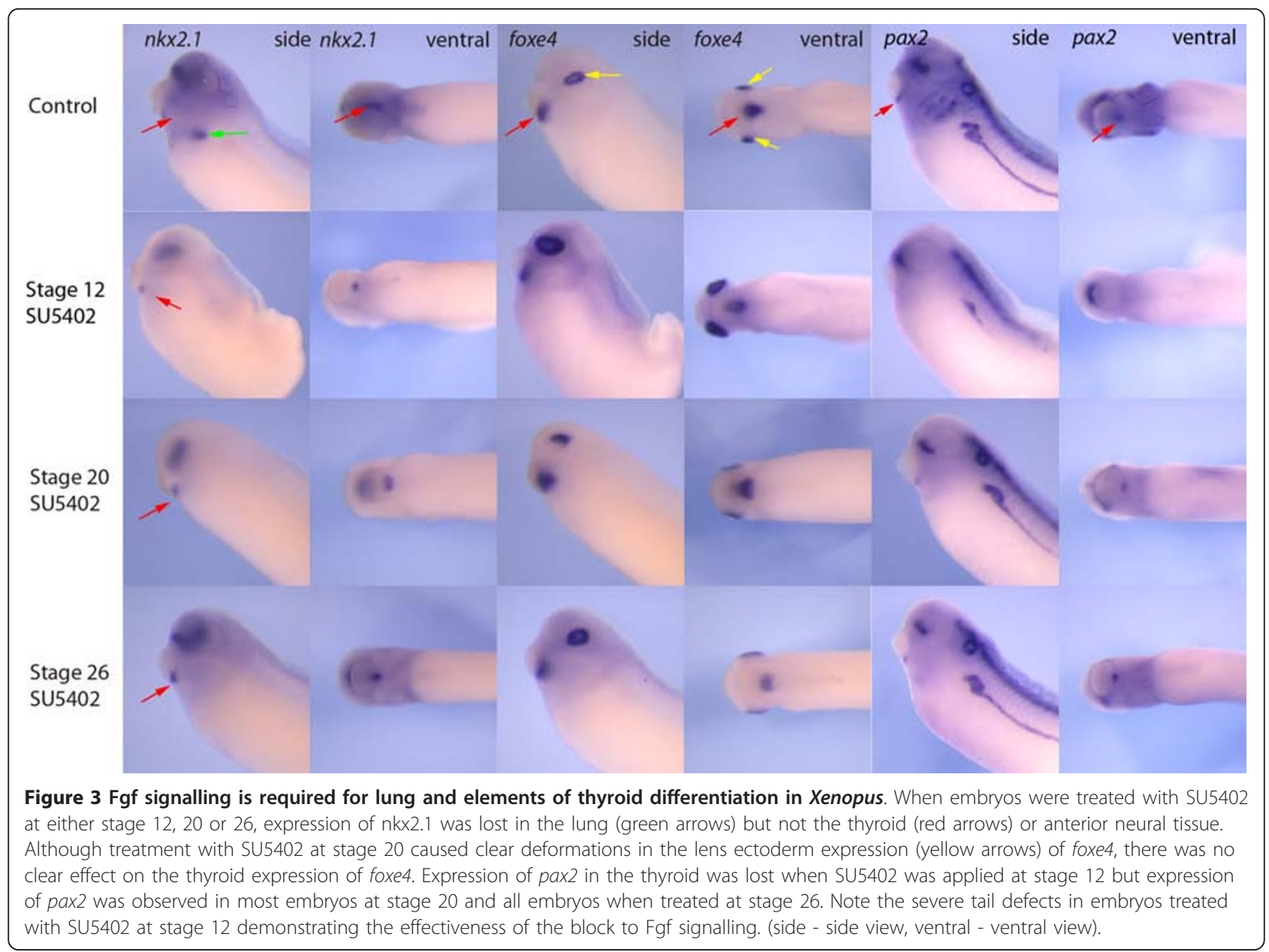

response to treatment with retinoic acid. As $n k x 2.1$ is expressed in both the early thyroid and lung, it was perhaps not surprising that there was no difference in expression of $n k x 2.1$ when embryos were exposed to RA or when RA signalling was blocked using the RA inhibitor at stage 26 (Figure 5). However, pax2 and foxe4, both markers of the early thyroid are not expressed in the thyroid in RA treated embryos, although expression at other sites is not lost in the same embryos. Foxe 1 has been described as another early marker of the thyroid [25] but we find that it does not have the same strong midline expression seen with other thyroid markers, although we cannot rule out low levels of expression.

Table 1 Presence of pax2 and sftpb expression in the presumptive thyroid exposed to different concentrations of RA

\begin{tabular}{lllll}
\hline & $\mathbf{1} \boldsymbol{\mu M}$ RA & $\mathbf{0 . 1} \boldsymbol{\mu M}$ RA & $\mathbf{0 . 0 1} \boldsymbol{\mu M}$ RA & DMSO control \\
\hline pax2 & $0 / 60$ & $32 / 61$ & $54 / 64$ & $54 / 54$ \\
sftpb & $74 / 83$ & $63 / 84$ & $37 / 81$ & $0 / 75$ \\
\hline
\end{tabular}

The numbers represent the number of embryos expressing the gene in the thyroid over the total number of embryos examined.
Nevertheless, RA had no discernable effect on expression of foxe 1 in that region although some patterning differences were noted in the pharyngeal region (Figure 5).

Hhex is a homeobox-containing transcription factor that is first expressed in a broad domain in the anterior endoderm and expression then becomes restricted to the forming thyroid and liver [1,31]. Exposure to retinoic acid during late gastrulation is known to eliminate hhex expression in the thyroid [16] and we wanted to ensure that our later retinoic acid treatments would still result in loss of thyroid expression. Treatment with RA at stage 26 was also able to eliminate the expression of hhex in the thyroid without loss of expression in the liver (Figure 6), further suggesting that RA blocks the full repertoire of early thyroid transcription factors. We also examined whether blocking RA signalling would change hhex expression. If RA signalling was blocked between stage 14 and 26, there was no obvious change in hhex expression in either the liver or thyroid (Figure 6).

Although addition of RA as late as stage 34 resulted in expression of $s f t p b$ in the presumptive thyroid, if the retinoic acid was added between stages 32 and 34, there 


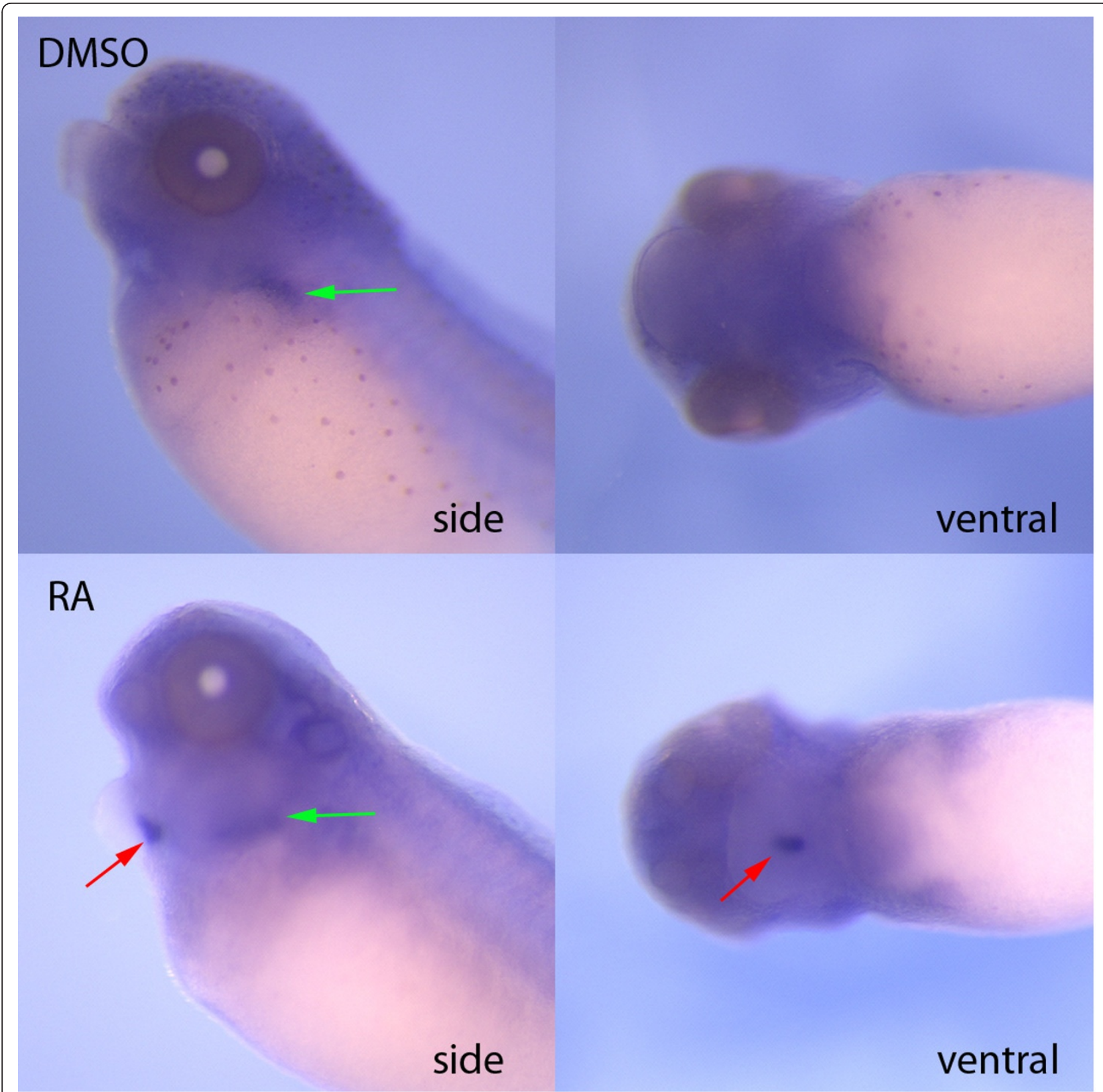

Figure 4 Exogenous retinoic acid causes ectopic expression of sftpc in the presumptive thyroid. Whole mount in situ hybridization for sftpc demonstrated that embryos treated at stage 26 with exogenous retinoic acid showed ectopic expression of sftpc in the developing thyroid (red arrows) as well as the normal expression in the lung (green arrows). (side - side view, ventral - ventral view).

was no loss of pax2 and foxe4 expression. This suggests that the tissue becomes ambiguous, expressing transcription factors that define it as thyroid in addition to the lung differentiation markers (Table 2).

One simple model for explaining differentiation of the thyroid might be that gastrulation movements position the presumptive thyroid far enough towards the rostral end of the embryo that it is not exposed to retinoic acid. If so, we would predict that simply transplanting the presumptive thyroid to the flank of the embryo, where there is robust aldh1a2 expression, may be sufficient to cause those precursors to differentiate as lung. This would also directly test whether endogenous levels of retinoic acid would be sufficient to cause the thyroid to lung switch. When presumptive thyroid explants were removed and cultured to stage 38 they did not express sftpb but robust expression of pax 2 could be seen in the cultured explants (Figure 


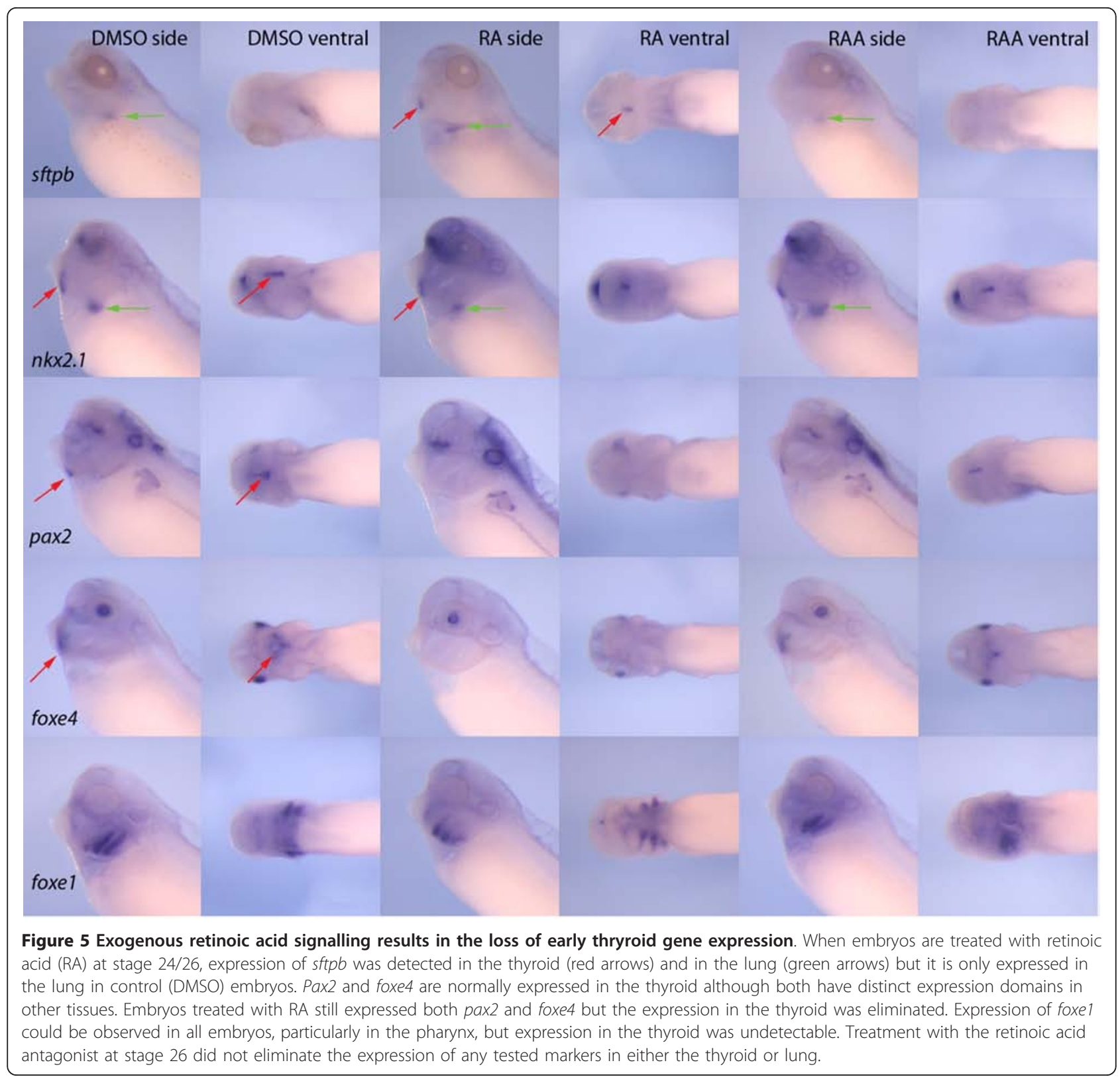

7A,B). When explants were transplanted into the flank of a donor embryo and allowed to heal, the explants could be seen to express sftpb (Figure 7C, E) suggesting that endogenous levels of RA can cause the presumptive thyroid to express sftpb. However, the transplants also continued to express pax2 suggesting that, similar to when RA is added between stages 30 and 34, the tissue has not lost all thyroid characteristics (Figure 7D,F). The size of the expression domain also corresponded well with the normal size of the thyroid suggesting that it was only the presumptive thyroid domain that was expressing sftpb.

\section{Discussion}

RA and FGF are required for lung development in Xenopus

Little is known about the signalling mechanisms required for lung development in Xenopus. A recent study has suggested that wnt and hedgehog signalling are needed for later lung development based on the expression patterns of shh, wht7b, wnt5a and wifl but their functional roles have yet to be elucidated. Here we show that both RA (Figure 2) and Fgf (Figure 3) signalling are necessary for differentiation of the lung in Xenopus. Both of these signalling systems are required for 


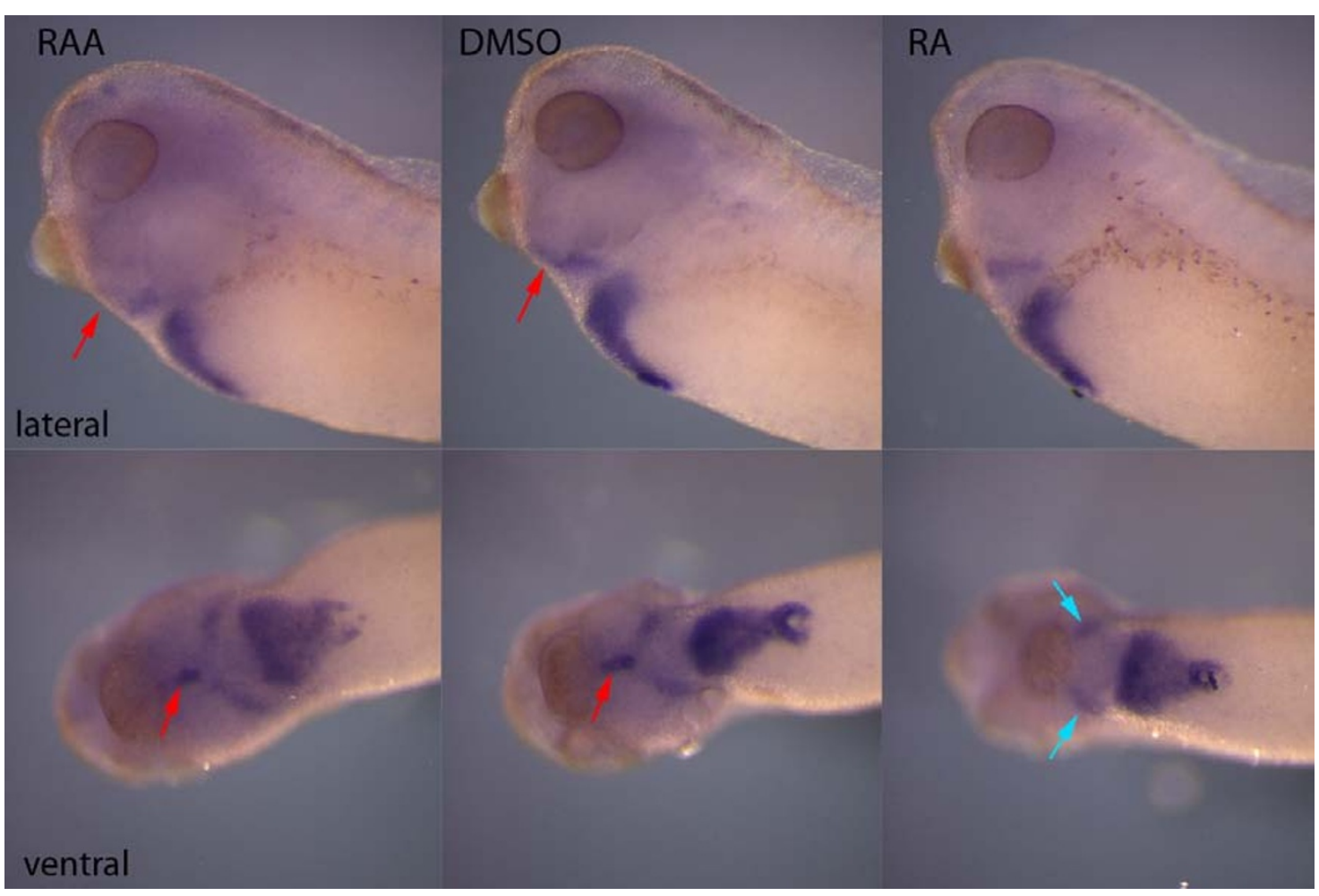

Figure 6 Treatment with retinoic acid eliminated expression of hhex in the thyroid but not liver. When embryos were treated at stage $24 / 26$ and cultured to stage 36 , expression could be detected near the thyroid even in RA treated embryos but in ventral view it can be clearly seen that midline expression of hhex (red arrow) is eliminated by RA but there is an additional region of expression lateral to the thyroid (blue arrows) that is not lost when embryos were treated with RA. There is also no obvious change in the expression of the large liver expression domain found caudal to the thyroid expression.

lung differentiation in the mouse and the two systems have been linked as loss of RA results in reduced levels of Fgf10 and subsequent lung agenesis [14]. It would appear that both RA and Fgf are very high in the

Table 2 Effect of RA on presence of sftpb, pax2 and foxe4 expression in specific organs

\begin{tabular}{lcccccc}
\hline & \multicolumn{2}{c}{ sttpb expression } & \multicolumn{2}{c}{ pax2 expression } & \multicolumn{2}{c}{ foxe4 expression } \\
Treatment & Lung & Thyroid & Thyroid & Kidney & Thryoid & Eye \\
\hline St. 14 DMSO & $33 / 33$ & $0 / 33$ & $10 / 10$ & $10 / 10$ & $11 / 11$ & $11 / 11$ \\
St. 14 RA & $32 / 32$ & $0 / 32$ & $0 / 11$ & $11 / 11$ & $0 / 10$ & $0 / 10$ \\
St. 26 DMSO & $82 / 82$ & $0 / 82$ & $70 / 70$ & $70 / 70$ & $50 / 50$ & $50 / 50$ \\
St. 26 RA & $81 / 82$ & $78 / 82$ & $0 / 74$ & $74 / 74$ & $0 / 54$ & $54 / 54$ \\
St. 30 DMSO & $22 / 22$ & $0 / 22$ & $12 / 12$ & $12 / 12$ & $9 / 12$ & $12 / 12$ \\
St. 30 RA & $24 / 26$ & $20 / 26$ & $2 / 11$ & $11 / 11$ & $2 / 11$ & $11 / 11$ \\
St. 32 DMSO & $42 / 42$ & $0 / 42$ & $26 / 26$ & $26 / 26$ & $24 / 24$ & $24 / 24$ \\
St. 32 RA & $39 / 40$ & $39 / 40$ & $21 / 25$ & $25 / 25$ & $23 / 23$ & $23 / 23$ \\
St. 34 DMSO & $6 / 6$ & $0 / 6$ & $10 / 10$ & $10 / 10$ & $28 / 28$ & $28 / 28$ \\
St. 34 RA & $5 / 5$ & $5 / 5$ & $10 / 10$ & $10 / 10$ & $16 / 16$ & $16 / 16$ \\
St. 36 DMSO & $6 / 6$ & $0 / 6$ & $10 / 10$ & $10 / 10$ & $10 / 10$ & $10 / 10$ \\
St. 36 RA & $6 / 6$ & $0 / 6$ & $10 / 10$ & $10 / 10$ & $10 / 10$ & $10 / 10$ \\
\hline
\end{tabular}

The numbers represent the number of embryos expressing the gene in the organ examined over the total number of embryos examined. hierarchy of lung specification as both are required well before the initial expression of $n k x 2.1$.

The requirement for RA signalling is lost quite early in the development of the lung. If the antagonist is added as early as stage 20, well before the expression of $n k x 2.1$, there is still detectable expression of $s f t p b$. If the antagonist is added at stage 26, the expression of sftpb is essentially the same as control embryos. The sftpb promoter in humans has binding sites for both retinoic acid receptors and for $\mathrm{nkx} 2.1$ and the two transcription factors act synergistically to drive expression of $s f t p b$ [32]. Although we cannot make any conclusions regarding the levels of $s f t p b$ expression using whole mount in situ hybridization, our results demonstrate that the activation of the retinoic acid receptors by RA is not a requirement for $s f t p b$ expression.

The requirement for Fgf also occurs early in Xenopus as blocking Fgf signalling at stage 20 causes a loss of $n k x 2.1$ expression, although unlike $\mathrm{RA}$, the requirement is maintained later as blocking at stage 26 still showed a complete loss of $n k x 2.1$ (Figure 3 ) and subsequent sftpb expression (Additional File 1, Figure S1) in the lung. 


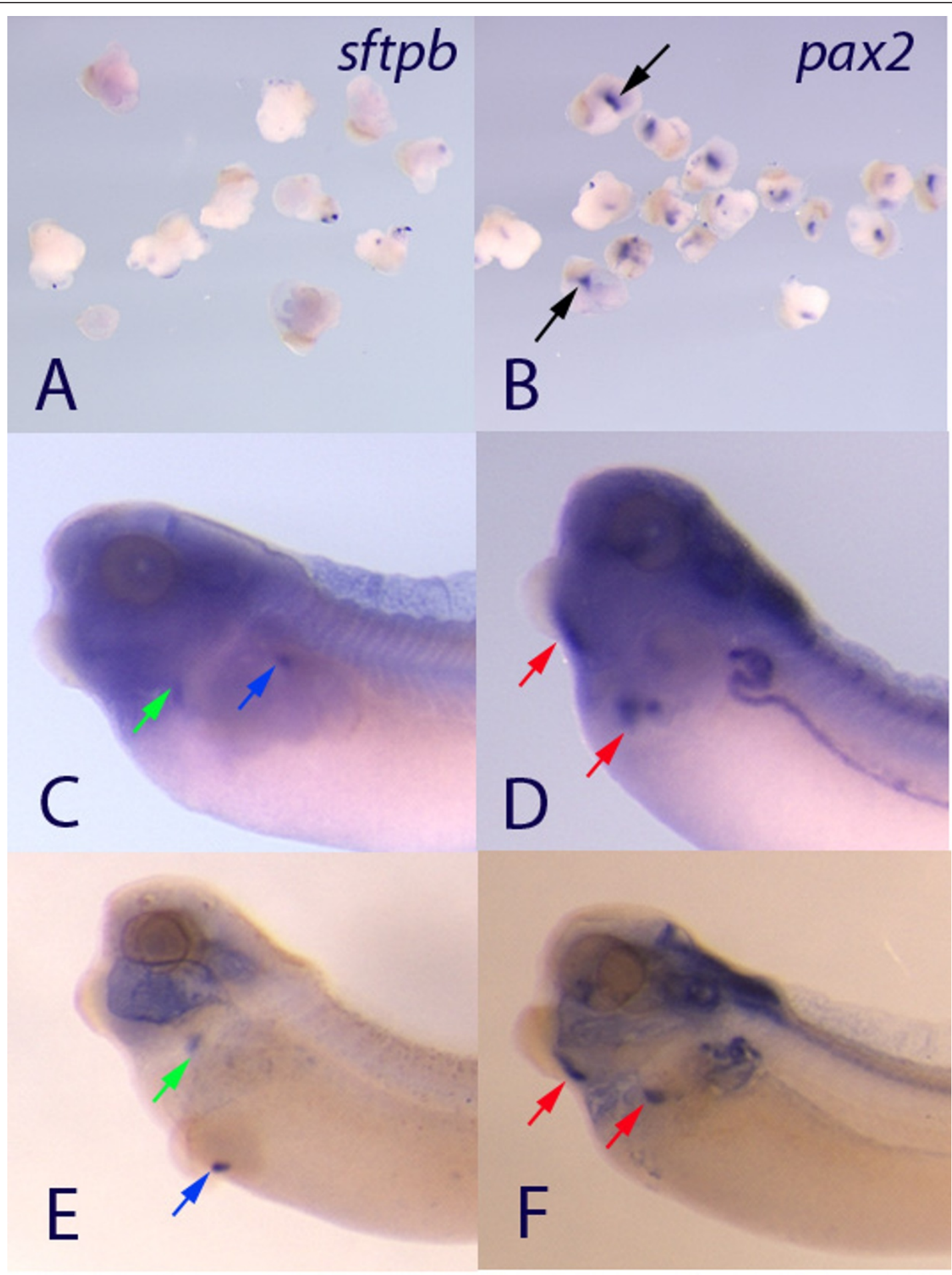

Figure 7 Transplantation of the presumptive thyroid explants into the embryo flank is sufficient to cause expression of sftpb in the explant. When explants of the presumptive thyroid region are cultured alone, they do not express sftpb (A) but do express pax2 (B - black

arrows). Note that the small blue flecks are seen on the surface of many embryos due to the stickiness of the cement gland that is also usually in part of the explant. When those same explants are placed on to the flank region immediately after they are removed from donor embryos, sftpb expression can be seen in the explant (blue arrows) as well as the endogenous lung (green arrows). When explants are transplanted, expression of pax2 (red arrows) can be seen in the explants. Embryos in C and D are normal views and embryos in $\mathrm{E}$ and $\mathrm{F}$ have been cleared to better visualize staining although cavity staining is also seen when embryos are cleared. 
Requirements of RA and Fgf signalling for development of the thyroid gland

When RA signalling was blocked, expression of thyroid markers was not affected (Figure 5). In mouse, Fgf8, regulated by tbx 1 and arising from the secondary heart field, is required for normal thyroid development although loss of fgf8 did not eliminate the thyroid [11]. In zebrafish, hand 2 is upstream of $f g f 8$ and this pathway is essential for thyroid development and again it is the cardiac mesoderm that is the source of the Fgf signal [7]. Thus it is surprising that the SU5402 treatments had little effect on expression of either $n k x 2.1$ or foxe4, although it was clear that SU5402 was able to cause developmental defects in the treated embryo, including loss of $n k x 2.1$ expression in the lung, severe loss of tail (Figure 3) and loss of sprouty 2 expression (Additional File 1, Figure S1).

We did find that the expression of pax2 in the thyroid was lost with early SU5402 treatments. The thyroid itself does not appear to be a direct target of the Fgf signal in zebrafish [7]. If the potential intermediate tissue were already established before we treated with SU5402 in Xenopus, this could provide an explanation for the lack of effect on $n k x 2.1$ and foxe 4 expression. Identification of the intermediate tissue is needed in order to test this possibility. The lack of pax 2 expression while $n k x 2.1$ and foxe 4 expression is maintained is perhaps expected. In pax8 knockout mice, the early thyroid primordium is maintained with normal $n k x 2.1$ expression although the thyroid is eventually lost at later stages due to apoptosis [5,33]. Thus, eventual loss of the thyroid might eventually be expected in Xenopus when Fgf signalling is blocked at very early stages.

\section{RA causes presumptive thyroid tissue to express lung- specific markers}

We have observed that addition of exogenous RA results in expression of $s f t p b$ and $s f t p c$ in the thyroid (Figure 2,4 ), both of which are clear markers of the differentiated lung. This does not seem to be simple ectopic expression as thyroid expression of hhex, pax2, and foxe 4 are lost with the addition of RA (Figure 5,6). Hhex, pax8, foxe 4 , and $n k x 2.1$ have been identified as forming a key transcription factor network for maintaining the thyroid [5] and it appears that a similar network exists in Xenopus. Pax2 substitutes for the expression of pax8 [6] and based on our expression data, it appears that foxe 4 is more closely related to the central thyroid region than foxe1 (Figure 5). Nevertheless, the early thyroid markers that we have examined are lost with the addition of RA suggesting that there has been a loss of the thyroid developmental program. The one exception is that expression of $n k x 2.1$ is maintained but it is also normally expressed in the lung. The suppression of hhex expression by exogenous RA has also been observed in the chick although in that system there was also loss of $n k x 2.1$ expression [13] suggesting that RA is sufficient to block thyroid development but not able to cause the fate switch that we observe in Xenopus. In the same chick study it was concluded that the thyroid must form in the absence or at very low levels of RA [13]. Our results confirm this and suggest that in Xenopus, the absence of RA is actually required for thyroid development, as presence of RA would result in potential differentiation as lung tissue.

We were not able to look at any differentiation markers of thyroid. Although thyroid hormone has been extensively studied in terms of metamorphosis in Xenopus, little is known about the ontogeny of the enzymes required for thyroid hormone production. A study examining the expression of thyroid peroxidase, type II idothyronine deiodinase, and type III iodothyronine deiodinase showed that none of these were expressed in the thyroid before stage 41 [34]. Interestingly, although initially described as thyroid gland expression, thyroid peroxidase is expressed in the lung at stage 43 [34] further demonstrating the close relationship between these two organs. We also hoped to determine whether RA-treated thyroid progenitors would form structures reminiscent of a mature lung such as branching or alveoli. However, these are very late events in Xenopus development [35] and embryos treated with RA usually developed swelling around the heart that did not allow culturing to later stages.

The recent finding that sfrp 5 and wnt 11 , both linked to the planar cell polarity pathway [36,37], are necessary for foregut specification suggests that morphogenetic movements of the endoderm may be important for proper specification of the different organ systems [38]. The requirement for morphogenesis suggests a relatively simple model whereby the early movements of the endoderm simply result in the furthest anterior endoderm, the presumptive thyroid, being beyond the mesodermal domains where retinoic acid is being synthesized. By simply physically moving the presumptive thyroid endoderm back to regions that would be predicted to have high levels of RA, the transplanted endoderm was able to express lung differentiation markers (Figure 7). However, we cannot rule out the possibility that other factors, present in the posterior embryo, might also be responsible for the transition.

\section{Conclusions}

Both RA and FGF signalling are required for the differentiation of the lung in Xenopus embryos. RA is required for lung development before the lung primordium expresses $n k x 2.1$ and $s f t p b$ can still be expressed in embryos that are treated with an RA antagonist in 
later development. If the presumptive thyroid is exposed to RA before stage 20 , early thyroid transcription factors are suppressed and lung differentiation markers will be expressed in the presumptive thyroid.

\section{Methods \\ Embryo Collection}

Female Xenopus laevis frogs were injected with 600-700 IU of human chorionic gonadotrophin (Sigma) to induce ovulation. In vitro fertilization of ovulated eggs was performed in $80 \%$ Steinberg's solution containing minced testis. Embryos were dejellied with $2.5 \%$ cysteine, $\mathrm{pH}$ 8.0, and cultured in 20\% Steinberg's solution. Embryos were staged according to the Nieuwkoop and Faber staging table [35].

\section{Cloning of sftpb and sftpc}

Adult Xenopus laevis lung cDNA was used to amplify both $s f t p b$ and $s f t p c$. The initial clones were amplified by polymerase chain reaction using the following sequences: $s f t p b$ forward 5'-cagtgggtccacaggatgac-3' and reverse 5'-gacggccccacactctttag-3' and sftpc forward 5'ggctgcacatgagtcaaaaac-3" and reverse 5'-ctgttccggatccatttgtg-3'. The sequence obtained from the $s f t p b$ amplicon was used to generate new primers for a 5 ' rapid amplification of cDNA ends procedure that gave a longer cDNA clone that included the start codon. Sequencing of the clone showed that it matched previously identified sftpb sequence (Genbank Ref. NM_001096917.1). The sftpc sequence matched the previously identified $s f t p c$ sequence (Genbank Ref NM_001096721.1).

\section{Embryo Treatments}

Embryos were treated with $1 \mu \mathrm{M}$ all-trans RA (Sigma) or $1 \mu \mathrm{M}$ pan RAR antagonist (Allergan \#193109) [30,39] in $20 \%$ Steinberg's solution at various stages depending on the experiment. Stock solutions for both RA and RAA were $1 \mathrm{mM}$ dissolved in DMSO, and therefore a control treatment was performed with $0.1 \%$ DMSO in $20 \%$ Steinberg's solution. Fgf signaling was inhibited by the

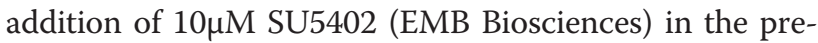
sence of $100 \mu \mathrm{M}$ ATP. Control treatments were DMSO and ATP alone.

Explants were removed at stage 18/20 using the cement gland as a guide. Explants were cut and maintained in $1 \times$ MBS. Explants were transplanted by creating a small wound in the recipient embryo at the site for transplantation and the transplantation was also conducted in $1 \times$ MBS. After the explant had healed, the embryos were transferred back to 20\% Steinberg's solution.

\section{In situ Hybridization}

Whole-mount in situ hybridizations were performed according to [40] with modifications: embryos were blocked in Tris buffered saline, 20\% heat treated sheep serum; and the Proteinase K, and RNase A steps were omitted. Antisense Dig-labelled riboprobes for $s f t p b$ and $s f t p c$ were generated by cutting the plasmids with $X b a 1$ and transcribing with SP6 RNA polymerase according to established protocols [40]. Antisense Dig-labelled riboprobes for pax2a [6], foxe1, foxe4, [25], nkx2.1 [24], and hhex [41] were also generated using the same methods. BM Purple (Roche Diagnostics) was used as the alkaline phosphatase substrate throughout and embryos were fixed for twenty minutes in MEMPFA before removing endogenous pigment in $1 \%$ hydrogen peroxide, $5 \%$ formamide, and $0.5 \% \mathrm{SSC}$ for several hours after the colour reaction. For some images, the embryos were cleared by immersion in 1 part benzyl alcohol: 2 parts benzyl benzoate after dehydration in methanol. Embryos were visualized using a Leica MZ12 dissecting microscope and images were taken using Northern Eclipse software (Empix Imaging; Mississauga, ON, Canada).

\section{Additional material}

Additional file 1: Treatment with SU5402 causes a loss of both sftpb and sprouty2 expression. Embryos treated with SU5402, in order to block Fgf signalling, do not express stpb indicating that there is a loss of differentiated lung. The location of the differentiated lung (green arrow) can be seen in the control embryo (DMSO treated). Expression of sprouty2 (spry2) was used to demonstrate the effectiveness of the Fgf signalling block. Sprouty2 is a target of Fgf signalling and strong expression can normally be seen at the midbrain-hindbrain border (yellow arrow) and in the pharynx (purple arrow). The expression of sprouty 2 in these regions is effectively eliminated by addition of SU5402 at all times tested. Treatment times are indicated at the top of each column (eg. T-st12 indicates that the treatment was initiated at embryonic stage 12).

\section{Acknowledgements}

We thank Dr. R. Chandraratna for providing the retinoic acid antagonist and advice on its use. We thank H. El-Hodori, A. Brandli, P. Krieg and A. Zorn for generously providing plasmids. Finally, we would like to thank anonymous reviewers for useful suggestions. This project was supported by grants awarded to TAD from the Canadian Institutes of Health Research (MOP74663) and from the National Sciences and Engineering Research Council (Canada). SJD was supported by a CIHR doctoral fellowship. Initial seed funding for the project came from Lawson Health Research Institute Internal Research Fund program.

\section{Author details}

${ }^{1}$ Children's Health Research Institute, London, Ontario, Canada. ${ }^{2}$ Department of Biology, University of Western Ontario, London, Ontario Canada. ${ }^{3}$ Department of Obstetrics and Gynecology, University of Western Ontario, Ontario, Canada. ${ }^{4}$ Department of Biochemistry, The University of Western Ontario, London, Ontario, N6A 5C1, Canada. ${ }^{5}$ Department of Paediatrics, Department of Physiology and Pharmacology, The University of Western Ontario, London, Ontario, Canada. 


\section{Authors' contributions}

JHW carried out the majority of experiments on the embryos. SJD did the analysis of Fgf requirements. NED did the analysis of hhex expression. LZ cloned the Xenopus stpb and sftpc sequences and characterized them. FP participated in the design and interpretation of experiments. TAD participated in the design and coordination of all experiments and prepared the draft manuscript. All authors read and approved the final manuscript

Received: 27 July 2011 Accepted: 20 December 2011 Published: 20 December 2011

\section{References}

1. Zorn AM, Wells JM: Vertebrate endoderm development and organ formation. Annu Rev Cell Dev Biol 2009, 25:221-51.

2. Dessimoz J, Opoka R, Kordich JJ, Grapin-Botton A, Wells JM: FGF signaling is necessary for establishing gut tube domains along the anteriorposterior axis in vivo. Mech Dev 2006, 123:42-55.

3. Kanai-Azuma M, Kanai Y, Gad JM, Tajima Y, Taya C, Kurohmaru M, Sanai Y, Yonekawa $\mathrm{H}$, Yazaki $\mathrm{K}$, Tam PP, et al: Depletion of definitive gut endoderm in Sox17-null mutant mice. Development 2002, 129:2367-79.

4. Chawengsaksophak K, de Graaff W, Rossant J, Deschamps J, Beck F: Cdx2 is essential for axial elongation in mouse development. Proc Natl Acad Sci USA 2004, 101:7641-5.

5. Parlato R, Rosica A, Rodriguez-Mallon A, Affuso A, Postiglione MP, Arra C, Mansouri A, Kimura S, Di Lauro R, De Felice M: An integrated regulatory network controlling survival and migration in thyroid organogenesis. Dev Biol 2004, 276:464-75.

6. Heller N, Brandli AW: Xenopus Pax-2/5/8 orthologues: novel insights into Pax gene evolution and identification of Pax-8 as the earliest marker for otic and pronephric cell lineages. Dev Genet 1999, 24:208-19.

7. Wendl T, Adzic D, Schoenebeck JJ, Scholpp S, Brand M, Yelon D, Rohr KB: Early developmental specification of the thyroid gland depends on hanexpressing surrounding tissue and on FGF signals. Development 2007, 134:2871-9.

8. Wan $\mathrm{H}$, Kaestner KH, Ang SL, Ikegami M, Finkelman FD, Stahlman MT, Fulkerson PC, Rothenberg ME, Whitsett JA: Foxa2 regulates alveolarization and goblet cell hyperplasia. Development 2004, 131:953-64.

9. Maeda Y, Dave V, Whitsett JA: Transcriptional control of lung morphogenesis. Physiol Rev 2007, 87:219-44.

10. Sherwood Rl, Chen TY, Melton DA: Transcriptional dynamics of endodermal organ formation. Dev Dyn 2009, 238:29-42.

11. Lania G, Zhang Z, Huynh T, Caprio C, Moon AM, Vitelli F, Baldini A: Early thyroid development requires a Tbx1-Fgf8 pathway. Dev Biol 2009, 328:109-17.

12. Serls AE, Doherty S, Parvatiyar P, Wells JM, Deutsch GH: Different thresholds of fibroblast growth factors pattern the ventral foregut into liver and lung. Development 2005, 132:35-47.

13. Bayha E, Jorgensen MC, Serup P, Grapin-Botton A: Retinoic acid signaling organizes endodermal organ specification along the entire anteroposterior axis. PLoS One 2009, 4:e5845.

14. Wang Z, Dolle P, Cardoso WV, Niederreither K: Retinoic acid regulates morphogenesis and patterning of posterior foregut derivatives. Dev Biol 2006, 297:433-45.

15. Desai TJ, Malpel S, Flentke GR, Smith SM, Cardoso WV: Retinoic acid selectively regulates Fgf10 expression and maintains cell identity in the prospective lung field of the developing foregut. Dev Biol 2004, 273:402-15.

16. Chen Y, Pan FC, Brandes N, Afelik S, Solter M, Pieler T: Retinoic acid signaling is essential for pancreas development and promotes endocrine at the expense of exocrine cell differentiation in Xenopus. Dev Biol 2004, 271:144-60.

17. Martin M, Gallego-Llamas J, Ribes V, Kedinger M, Niederreither K, Chambon P, Dolle P, Gradwohl G: Dorsal pancreas agenesis in retinoic acid-deficient Raldh2 mutant mice. Dev Biol 2005, 284:399-411.

18. Chen Y, Pollet N, Niehrs C, Pieler T: Increased XRALDH2 activity has a posteriorizing effect on the central nervous system of Xenopus embryos. Mech Dev 2001, 101:91-103.

19. Lynch J, McEwan J, Beck CW: Analysis of the expression of retinoic acid metabolising genes during Xenopus laevis organogenesis. Gene Expr Patterns 2011, 11:112-7.
20. Lipscomb K, Schmitt C, Sablyak A, Yoder JA, Nascone-Yoder N: Role for retinoid signaling in left-right asymmetric digestive organ morphogenesis. Dev Dyn 2006, 235:2266-75.

21. Hyatt BA, Resnik ER, Johnson NS, Lohr JL, Cornfield DN: Lung specific developmental expression of the Xenopus laevis surfactant protein $C$ and B genes. Gene Expr Patterns 2007, 7:8-14.

22. Zuo YY, Veldhuizen RA, Neumann AW, Petersen NO, Possmayer F: Current perspectives in pulmonary surfactant-inhibition, enhancement and evaluation. Biochim Biophys Acta 2008, 1778:1947-77.

23. Tata JR: Amphibian metamorphosis as a model for the developmental actions of thyroid hormone. Mol Cell Endocrinol 2006, 246:10-20.

24. Small EM, Vokes SA, Garriock RJ, Li D, Krieg PA: Developmental expression of the Xenopus Nkx2-1 and Nkx2-4 genes. Mech Dev 2000, 96:259-62.

25. El-Hodiri HM, Seufert DW, Nekkalapudi S, Prescott NL, Kelly LE, Jamrich M: Xenopus laevis FoxE1 is primarily expressed in the developing pituitary and thyroid. Int I Dev Biol 2005, 49:881-4.

26. Chen F, Desai TJ, Qian J, Niederreither K, Lu J, Cardoso WV: Inhibition of Tgf beta signaling by endogenous retinoic acid is essential for primary lung bud induction. Development 2007, 134:2969-79.

27. Min H, Danilenko DM, Scully SA, Bolon B, Ring BD, Tarpley JE, DeRose M, Simonet WS: Fgf-10 is required for both limb and lung development and exhibits striking functional similarity to Drosophila branchless. Genes Dev 1998, 12:3156-61.

28. Sekine K, Ohuchi H, Fujiwara M, Yamasaki M, Yoshizawa T, Sato T, Yagishita N, Matsui D, Koga $Y$, Itoh N, et al: Fgf10 is essential for limb and lung formation. Nat Genet 1999, 21:138-41.

29. Chen F, Cao Y, Qian J, Shao F, Niederreither K, Cardoso WV: A retinoic aciddependent network in the foregut controls formation of the mouse lung primordium. J Clin Invest 2010, 120:2040-8.

30. Teng M, Duong $T$, Johnson AT, Klein ES, Wang L, Khalifa B, Chandraratna RA: Identification of highly potent retinoic acid receptor alpha-selective antagonists. J Med Chem 1997, 40:2445-51.

31. Grapin-Botton A: Antero-posterior patterning of the vertebrate digestive tract: 40 years after Nicole Le Douarin's PhD thesis. Int I Dev Biol 2005, 49:335-47.

32. Naltner A, Ghaffari M, Whitsett JA, Yan C: Retinoic acid stimulation of the human surfactant protein B promoter is thyroid transcription factor 1 site-dependent. J Biol Chem 2000, 275:56-62.

33. Mansouri A, Chowdhury K, Gruss P: Follicular cells of the thyroid gland require Pax8 gene function. Nat Genet 1998, 19:87-90.

34. Tindall AJ, Morris ID, Pownall ME, Isaacs HV: Expression of enzymes involved in thyroid hormone metabolism during the early development of Xenopus tropicalis. Biol Cell 2007, 99:151-63.

35. Nieuwkoop PD, Faber J: Normal table of Xenopus laevis (Daudin): a systematical and chronological survey of the development from the fertilized egg till the end of metamorphosis. New York: Garland Pub; 1994.

36. Satoh W, Matsuyama M, Takemura H, Aizawa S, Shimono A: Sfrp1, Sfrp2, and Sfrp5 regulate the $\mathrm{Wnt} /$ beta-catenin and the planar cell polarity pathways during early trunk formation in mouse. Genesis 2008, 46:92-103.

37. Roszko I, Sawada A, Solnica-Krezel L: Regulation of convergence and extension movements during vertebrate gastrulation by the Wnt/PCP pathway. Semin Cell Dev Biol 2009, 20:986-97.

38. Li Y, Rankin SA, Sinner D, Kenny AP, Krieg PA, Zorn AM: Sfrp5 coordinates foregut specification and morphogenesis by antagonizing both canonical and noncanonical Wnt11 signaling. Genes Dev 2008, 22:3050-63.

39. Collop AH, Broomfield JA, Chandraratna RA, Yong Z, Deimling SJ, Kolker SJ, Weeks DL, Drysdale TA: Retinoic acid signaling is essential for formation of the heart tube in Xenopus. Dev Biol 2006, 291:96-109.

40. Harland RM: In situ hybridization: an improved whole-mount method for Xenopus embryos. Methods Cell Biol 1991, 36:685-95.

41. Zorn AM, Mason J: Gene expression in the embryonic Xenopus liver. Mech Dev 2001, 103:153-7.

doi:10.1186/1471-213X-11-75

Cite this article as: Wang et al: Retinoic acid is a key regulatory switch determining the difference between lung and thyroid fates in Xenopus laevis. BMC Developmental Biology 2011 11:75. 